\title{
Biologically inspired visual odometer for navigation of a flying robot
}

\author{
Fumiya Iida \\ Artificial Intelligence Laboratory, Department of Information Technology, University of Zurich, \\ Andreasstrasse 15, 8050 Zurich, Switzerland
}

\begin{abstract}
Experimental research in biology has uncovered a number of different ways in which flying insects use cues derived from optical flow for navigational purposes, such as safe landing, obstacle avoidance and dead reckoning. In this study, we use a synthetic methodology to gain additional insights into the navigation behavior of bees. Specifically, we focus on the mechanisms of course stabilization behavior and visually mediated odometer by using a biological model of motion detector for the purpose of long-range goal-directed navigation in $3 \mathrm{D}$ environment. The performance tests of the proposed navigation method are conducted by using a blimp-type flying robot platform in uncontrolled indoor environments. The result shows that the proposed mechanism can be used for goal-directed navigation. Further analysis is also conducted in order to enhance the navigation performance of autonomous aerial vehicles.
\end{abstract}

(C) 2003 Elsevier B.V. All rights reserved.

Keywords: Biorobotics; Aerial robotics; Visual navigation; Visual odometer; Optical flow

\section{Introduction}

The ability to navigate in a complex environment is crucial for both animals and robots. Particularly flying insects are capable of surprisingly good navigation despite the small size and relative simplicity of their brains. Biological studies of flying insects have revealed that the animals are largely relying on visual sensory inputs for their navigation. The vision systems of flying insects are exquisitely sensitive to motion, because visual motion induced by ego-motion can tell the animal much about its own motion and also about the structure of its environment. Behavior experiments with flies and bees show a number of different ways in which insects use cues derived from optical flow for navigational purposes (for review, see [10]). Early

E-mail address: iida@ifi.unizh.ch (F. Iida).

URL: http://www.ifi.unizh.ch/ailab/people/iida/. studies showed that a tethered fly inside a striped drum tends to turn in the direction in which the drum is rotated [8]. This reaction, the so-called optomotor response, serves to help the insect maintain a straight course by compensating for undesired deviations. In addition to such a simple reactive behavior, mechanisms for long-distance navigation are also crucial for such insects as honeybees, which navigate accurately and repeatedly from their hive to a food source. This type of navigation has been known to require that both directional and distance information be available. For the direction estimation, there is a lot of evidence that celestial cues, especially the polarization pattern of the sky play a crucial role [4], and recent studies of bees' behavior suggested that the amount of image motion plays an important role to estimate the distance traveled [12].

Inspired by these insect studies, the basic reactive behaviors observed in the animals, e.g. obstacle 
avoidance and fixation behaviors, have been modeled and implemented into many land-based robotic platforms (e.g. $[3,5,9,11])$; simulated flying agents were used for altitude control and obstacle avoidance [7]; a robotic gantry demonstrated the landing behavior of flies [13]; a "tethered" autonomous helicopter demonstrated its altitude control using optical flow [6]. A number of aerial robotic applications which employ optical flow-based controls have also been extensively explored (e.g. [1,14]). One of the interesting contrasts between the navigation of these robotics research and those of insects is that, whereas artificial aerial vehicles generally strongly rely on a number of different sensory modalities such as gyros, accelerometer, and GPSs, insects largely depend on vision with a variety of different processing pathways, from the low-level flight stabilization to the high-level long-distance navigation as mentioned above. Toward the comprehensive understanding of flying insects' navigation, which can be applicable for long-distance navigation of aerial autonomous vehicles, in this paper, we propose a method of goal-directed aerial navigation by exclusively using an embedded omni-directional visual sensor with optical flow. In order to evaluate the performance of the method, the flight experiments with an autonomous flying robot is presented. By comparing the experimental results with the biological experiments and the other robotic navigation studies, we discuss a number of different solutions in order to enhance the performance of the proposed navigation method.

In the following sections, the course stabilization and visual odometer models are proposed (Section 2) and we evaluate the performance in the navigation of a freely flying robot (Section 3). Further issues to enhance the proposed navigation capability are discussed in Section 4.

\section{Navigation models of a flying robot}

On the basis of the biological studies, this section explains models of course stabilization and visual odometer for the flying robot we have developed. These two models will be used for the experiments of goal-directed navigation in the later section.

\subsection{Course stabilization model}

Inspired by the flying insects' optomotor response, here we describe a course stabilization model for our flying robot. For the optical flow measurement, we employ a biologically plausible model of motion detection, the so-called elementary motion detector (EMD) or the Reichardt detector, shown in Fig. 1 (for review, see [2]). Two adjacent photoreceptors send their outputs to temporal high-pass filters that remove constant illumination containing no motion information. These

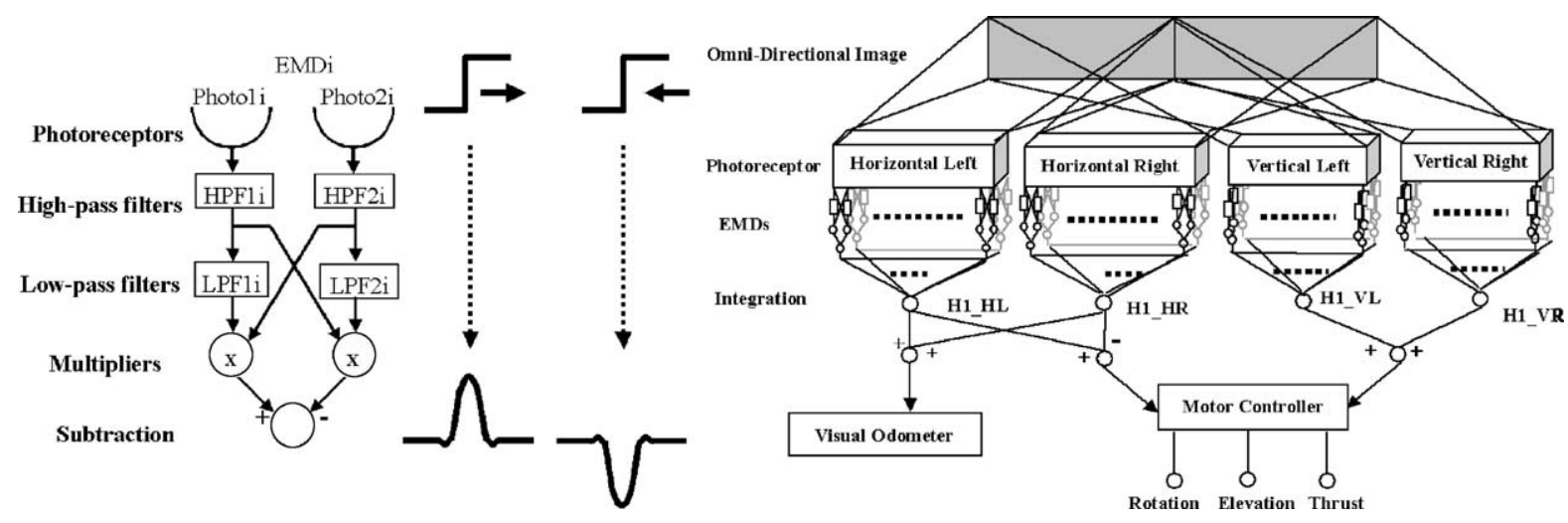

Fig. 1. Left: the Reichardt model of elementary motion detection. Photoreceptors, high-pass filters, low-pass filters, multipliers, and the subtraction module are wired in series. The output of the last step (subtraction) is an estimate of direction-sensitive image speed. Right: the controller circuit for the flying robot and visual odometer. The gray rectangle denotes the panoramic image extracted from the omni-directional camera on the robot. The image is given to both horizontally and vertically arranged photoreceptor arrays of EMDs. After the integrators and comparator, the outputs are given to motor controller and visual odometer modules. 
signals are then "delayed" by exploiting the phase lag inherent in a first-order temporal low-pass filter. Delayed channels are then correlated with adjacent, non-delayed channels by means of a multiplication operation. Finally the outputs of two opponent EMDs are subtracted to yield a direction-sensitive response. Although the nature of the neural mechanisms and the location in the visual pathway remains to be elucidated, some behaviors of the motion sensitive neurons in insects can be well characterized by this motion detector model [10]. The salient properties of the movement-sensitive mechanism underlying these responses are that it is directional, and that it does not encode the pure speed of the moving image, rather it is sensitive to the temporal frequency of intensity fluctuations generated by the moving image, and therefore confounds the speed of the image with its spatial structure. Fig. 1 illustrates the control procedure: at first, image from the panoramic vision system (see next section for the details) is log-polar transformed (the gray rectangle in Fig. 1), the intensity information of which is given to four two-dimensional EMD arrays, i.e. horizontal left (HL), horizontal right (HR), vertical left (VL), and vertical right (VR). These arrays extract horizontal motion and vertical motion from the left and the right lateral images. The outputs of each EMD array are then integrated in order to simulate the wide field motion sensitive interneurons $\mathrm{H} 1$ as follows:

$$
\begin{aligned}
& \mathrm{H} 1 \_\mathrm{HL}(t)=\sum_{i} \sum_{j} \mathrm{EMD}_{j} \mathrm{HL}_{i j}(t), \\
& \mathrm{H} 1 \_\mathrm{HR}(t)=\sum_{i} \sum_{j} \mathrm{EMD}_{j} \mathrm{HR}_{i j}(t) .
\end{aligned}
$$

To estimate the rotational ego-motion of the robot, the right and left horizontal motions are compared by a simple subtraction:

$S_{\text {rot }}(t)=\mathrm{H} 1 \_\mathrm{HL}(t)-\mathrm{H} 1 \_\mathrm{HR}(t)$.

The vertical motion, i.e. changes in height, is also estimated in a similar manner. The vertically arranged EMDs in the two-dimensional EMD arrays give the estimate of vertical motion. The outputs of these EMDs are integrated as $S_{\text {alt }}$ :

$$
\begin{aligned}
S_{\text {alt }}(t)= & \sum_{i} \sum_{j} \text { EMD_VL }_{i j}(t) \\
& +\sum_{i} \sum_{j} \text {EMD_VR }_{i j}(t) .
\end{aligned}
$$

These rotation and height sensory information induced by ego-motion is then given to a motor controller module. The three parameters are required for the control of the flying robot (see the next section for details), i.e. rotation, elevation and thrust motor outputs, $M_{\mathrm{R}}$, $M_{\mathrm{E}}$ and $M_{\mathrm{T}}$, respectively, which are determined by the following equation:

$$
\left[\begin{array}{c}
M_{\mathrm{R}} \\
M_{\mathrm{E}} \\
M_{\mathrm{T}}
\end{array}\right]=\left[\begin{array}{ccc}
W_{\mathrm{RS}} & 0 & 0 \\
0 & W_{\mathrm{ES}} & 0 \\
0 & 0 & W_{\text {bias }}
\end{array}\right] \cdot\left[\begin{array}{c}
S_{\text {rot }} \\
S_{\text {alt }} \\
1
\end{array}\right] .
$$

In this motor controller, $W_{\mathrm{RS}}$ and $W_{\mathrm{ES}}$ are heuristically determined so that $S_{\text {rot }}$ and $S_{\text {alt }}$ should be minimized, and $W_{\text {bias }}$ is set such that thrust motors are driven at constant speed.

\subsection{Visual odometer model}

The same horizontal EMD arrays that are used for course stabilization control are again used for the visual odometer (Fig. 1). Since the EMD response provides an angular velocity signal, the distance traveled could be estimated by integrating the EMD outputs over time. The responses from both right and left horizontal EMD arrays are integrated every time-step, which is given to the visual odometer module, then accumulated over time. Namely the visual odometer response, VO, is calculated by integrating the H1_HL, H1_HR output over time:

$$
\mathrm{VO}=\sum_{t}\left(\mathrm{H} 1 \_\mathrm{HR}(t)+\mathrm{H} 1 \_\mathrm{HL}(t)\right) .
$$

\section{Experiments}

This section presents a series of navigation experiments by using an autonomous flying robot. The models explained in the previous section are implemented in the robot, and then tested in uncontrolled indoor environments.

\subsection{Method}

To evaluate the performance of our model, we developed an autonomous flying robot, shown in Fig. 2. The flying robot Melissa is a blimp-like flying robot, which 


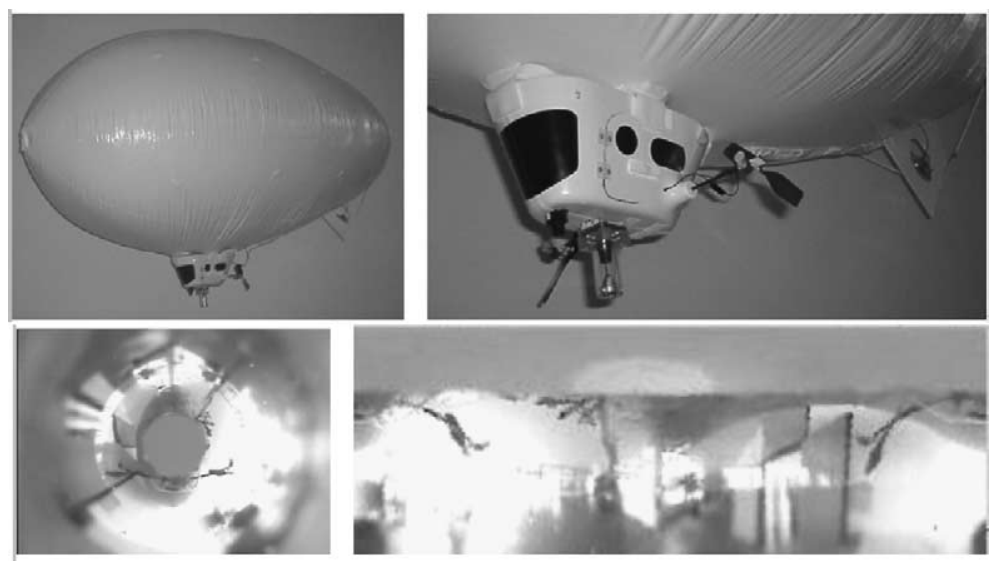

Fig. 2. Top: the autonomous flying robot, Melissa and its gondola, on the bottom of which a miniature panoramic camera is attached. Bottom: an image obtained by the panoramic vision system (left) and its log-polar transformed image (right), which is used in the experiments.

consists of a helium balloon, a gondola hosting the onboard electronics, and a host computer. The balloon is $2.3 \mathrm{~m}$ long and has a lift capacity of approximately $500 \mathrm{~g}$. Inside the gondola, there are three motors for elevation and thrust control (an additional motor for rotation control is attached directly to the balloon), a four-channel radio link, a miniature panoramic vision system, and batteries. The panoramic mirror has a hyperbolic surface that provides a visual field of $360^{\circ}$ on the horizontal plane and $130^{\circ}$ vertically. The control process of Melissa can be decomposed into three basic steps. First, the video signal from the CCD camera attached to the gondola is transmitted to the host computer via a wireless video link. Second, the images are then digitized on the host computer, which also performs the image processing in order to determine the target motor command. Third, the motor command is sent to the gondola also via radio transmission. The frame rate of the visual processing is set to a constant speed of $10.0 \mathrm{fps}$. The robot uses 180 (horizontal) $\times 60$ (vertical) pixels $(90 \times 30$ EMDs), which cover $360^{\circ}$ along horizontal plane and $120^{\circ}$ along vertical plane in the panoramic image homogeneously, i.e. angular distances between photoreceptors of the EMDs, the sampling bases, are $1.0^{\circ}$.

The W matrix of Eq. (5) is heuristically determined before experiments, since it is strongly dependent on the hardware conditions (floating balance of the robot, etc.) and environmental factors (air currents, etc.). Three sets of experiments are conducted in three different uncontrolled indoor locations (EXP 0-2 shown in Fig. 3), where we installed two video cameras to track and record the absolute trajectories of the robot for later analysis (Fig. 3, left). The experiments in
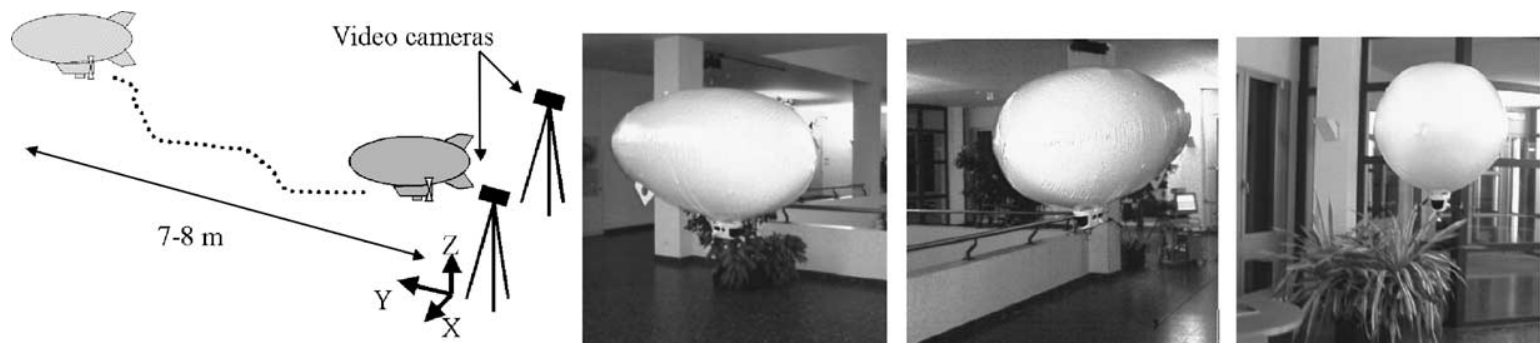

Fig. 3. Left: experimental setup for the navigation experiments. The experiments start with the same initial conditions, i.e. initial robot position and orientation. Two video cameras are installed to record the absolute trajectory of the robot for later analysis. Right: pictures of three different uncontrolled environments, which are tested in the experiments (EXP $0-2$, from left to right). 

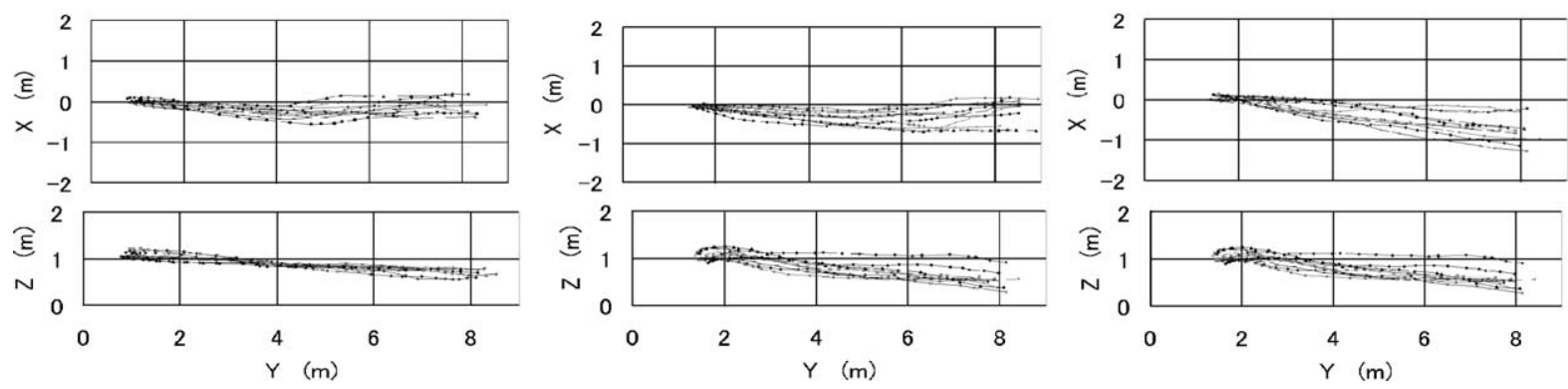

Fig. 4. 3D trajectories of the flying robot during the experiments. Each plot is extracted from the images recorded with the stereo video camera. The plots denote the position of the robot at $1 \mathrm{~s}$ time-step, and each graph contains the trajectories of 10 trials (EXP 0-2, from left to right).

each location consist of 10 flight trials: in one trial, the robot starts controlling at the same initial conditions, i.e. initial positions and initial orientations, and stops the operation after the same time duration. Due to the limitation of the experimental environments, the time duration is set to $25 \mathrm{~s}$.

\subsection{Results}

In Fig. 4, the plots show 3D trajectories of the robot. From the stereo video camera images recorded externally, absolute positions of the robot are extracted by tracking a fixed point of the robot in $1 \mathrm{~s}$ step. In these graphs, each trial starts from left toward right. Most of the trajectories in these graphs show that the robot maintains straight routes by balancing the right and left lateral image speeds. However, a small difference in the initial orientations of the robot results in relatively large deviations at the goal position, as shown in the $X-Y$ plane figure of EXP 2 in particular. For the height control, the robot tends to lose the height at the beginning of experiments, but eventually maintains the height in a certain range in all of the trials.

Fig. 5 shows the visual odometer responses that the robot experienced during each trial. The proposed visual odometer model measures almost the same distances in each experimental setup, EXP 0-2. Especially in EXP 0 and 1, the curve profiles of the visual odometer values over 10 trials show the similarity in their accumulation patterns, which indicates that the proposed visual odometer could potentially be capable of measuring the traveling distance. The relatively large deviations in EXP 2 could be due to the course deviation of the robot route trajectories as shown in
Fig. 4. Further issues will be discussed in the next section.

In addition, in Fig. 5, the visual odometer responses are plotted against the corresponding actual distances the robot travels. The actual distances in this figure are estimated from the externally observed 3D trajectories shown in Fig. 4. Despite the same flight duration of $25 \mathrm{~s}$, the variance of the visual odometer measurement is relatively large. However, the distributions from EXP 0 and 1 tend to monotonously increase as the actual distances increase. The result from EXP 2 seems to be, again, caused by the route deviation. From these experimental results, the proposed model is potentially capable of measuring the travelling distances visually, however, the measurement prone to depend on the route the robot experiences.

Further analyses are conducted with respect to the effects of the number of EMDs. In these analyses, from the stored data of EMD outputs, we re-calculated the visual odometer outputs by using the different numbers of EMDs: 1, 10, 20, 30, and 45 EMDs are selected over each left and right laterally distributed EMDs; then integrated their outputs over time. These results are normalized by the corresponding actual distances, and their standard deviations (S.D.s) are calculated as percent of mean values (Fig. 6). Therefore, when a S.D. results in a smaller value, it indicates that the visual odometer with the corresponding number of EMDs contains smaller error. There are two implications that should be mentioned from this analysis. Firstly, in principle, one EMD is essentially the minimum requirement of the proposed visual odometer model, although redundancy improves the measurement errors for all EXP 0-2. Secondly, these 

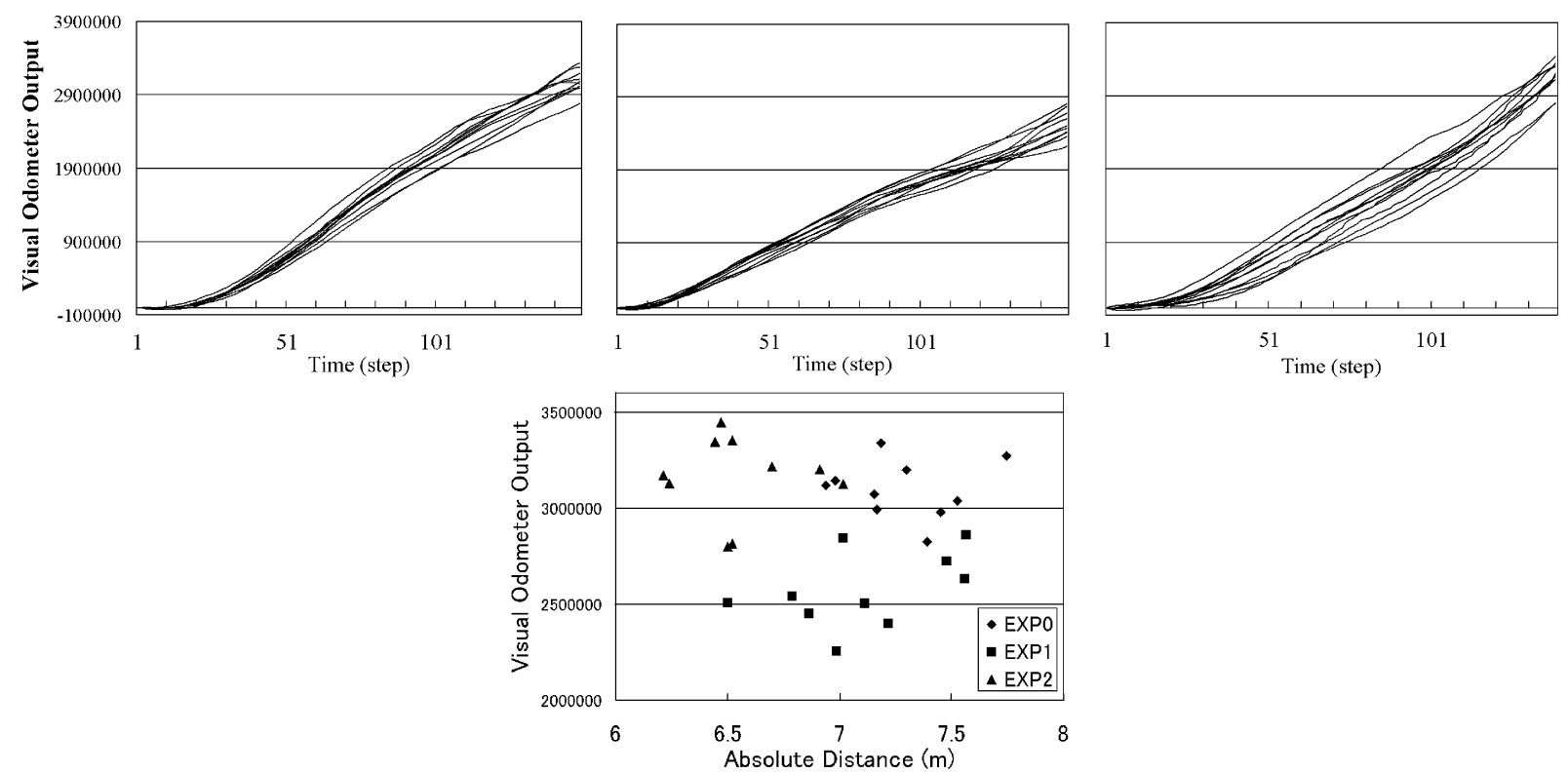

Fig. 5. Top: visual odometer responses of the 10 trials in the three experimental setups. The curve profiles show that the visual odometer accurately estimates the distances in the same experimental setup, particularly in EXP 0 and 1. Bottom: visual odometer measurement vs. actual distance. The actual distances that the robot traveled in each trial are extracted from Fig. 4.
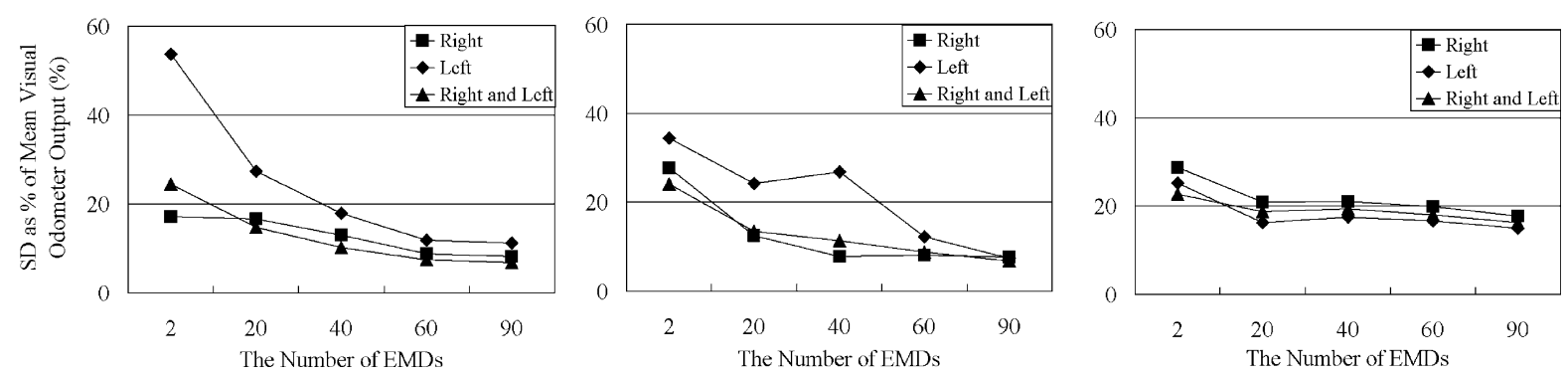

Fig. 6. The effect of the number of EMDs by S.D.s as percent of the mean values in visual odometer measurement. The graphs show that the number of EMDs changes the accuracy of visual odometer measurements.

results indicate that the performance of the proposed visual odometer could be improved by adaptively choosing a preferable visual field. For example, in EXP 0 and 1, the S.D.s of the left visual field are significantly larger than those of right, especially in the smaller numbers of EMDs. Therefore, in order to improve the performance, the robot should use the left visual field for the odometer. More generally, if the robot would have a task in which it should visit repeatedly the same goal location, the robot could adaptively choose/learn the appropriate number and positions of EMDs in order to improve the odometer performance.

\section{Discussion}

Given the experimental results, in this section, we discuss further issues of the proposed navigation model and possible solutions to improve the performance of the goal-directed navigation. 
For flight stabilization using optical flow, the robot has to visually extract the translation and rotation motions of its own body and compensate for them. While a flat visual projection area with a narrow view angle, such as a standard camera view, has a great disadvantage for this problem, the panoramic vision system makes the problem easier as employed in our experiments, where we compared the mean motion direction at the contralateral part of the panoramic image for the yaw rotational control. Furthermore, if necessary, the same principle can be applicable for the roll and pitch controls in the case of flying insects or the other robotic platforms with an omni-directional vision. For the altitude controls, in this paper, we employed the vertical EMDs to measure the vertical image movement. Most probably pure image speed detectors, rather than spatio-temporal filter such as EMDs which have non-linear responses with respect to image speed, would improve the performance of altitude control. Alternatively, measuring the apparent ground speed could be another solution to control altitude as demonstrated in [6]. The advantage of this solution is that the similar mechanism could be also used for safe landing [13].

Although the experimental results showed that the combination of course stabilization behavior and visual odometer could be used for goal-directed navigation, there are some potential solutions to improve the accuracy of the proposed visual odometer model. Since the performance of the visual odometer relies on the spatial structure experienced during the navigation as shown in Fig. 5, minimizing route deviations could improve the accuracy. Sensory feedback from compass information, for instance, is desirable to maintain a straight route, considering that celestial cues play an important role as a global compass in natural systems. Landmark navigation could also be a biologically plausible alternative solution to enhance the performance of course stabilization. In another solution, as suggested in Fig. 6, the EMD locations and the number of EMDs in the visual field need to be carefully considered for the better performance of the visual odometer, especially in unstructured environments. Considering that bees increase the accuracy of goal-directed navigation after learning phase, an adaptive learning mechanism in visual odometer model is expected. Alternatively, visual odometer could be based on a pure speed detector, rather than spatio-temporal fil- ters. However, as long as the robot follows the same route repeatedly, a spatio-temporal filter can be also usable.

\section{Conclusion}

In this paper, inspired by the navigation mechanisms of bees, we propose a method for goal-directed aerial navigation exclusively using a panoramic vision system. We performed experiments using a blimp-type robotic platform in unstructured indoor environments, and the combination of course stabilization and visual odometer models successfully demonstrates goal-directed navigation in the $3 \mathrm{D}$ indoor environments. In addition, the further analysis leads to potential solutions to enhance the navigation capability of the proposed models. Although the control of our blimp-type robotic platform is by far simpler than those of other platforms such as helicopters, it is expected to realize more sophisticated controls in more demanding situations with a vision-based architecture, as the natural evolution has found a solution for flying insects.

\section{Acknowledgements}

This work is supported by the Swiss National Science Foundation, grant no. 2000-061372.00, and the Swiss Federal Office for Education and Science (VIRGO TMR network, BBW no. 96.0148).

\section{References}

[1] O. Amidi, T. Kanade, K. Fujita, A visual odometer for autonomous helicopter fight, in: Y. Kakazu, M. Wada, T. Sato (Eds.), Intelligent Autonomous Systems, IOS Press, Amsterdam, 1998, pp. 123-130.

[2] A. Borst, M. Egelhaaf, in: F.A. Miles, J. Wallman (Eds.), Detecting Visual Motion: Theory and Models, Visual Motion and its Role in the Stabilization of Gaze, Elsevier, Amsterdam, 1993, pp. 3-27.

[3] N. Franceschini, J.M. Pichon, C. Blanes, From insect vision to robot vision, Philosophical Transactions of the Royal Society of London Series B 337 (1992) 283-294.

[4] J.L. Gould, C.G. Gould, The Honey Bee, Scientific American Library, New York, 1988.

[5] S.A. Huber, M.O. Franz, H.H. Bülthoff, On robots and flies: Modeling the visual orientation behavior of flies, Robotics and Autonomous Systems 29 (1999) 227-242. 
[6] T. Netter, N. Franceschini, A robotic aircraft that follows terrain using a neuromorphic eye, in: Proceedings of the 2002 IEEE/RSJ International Conference on Intelligent Robots and Systems, Lausanne, Switzerland, 2002, pp. 129-134.

[7] T.R. Neumann, H.H. Bülthoff, Insect inspired visual control of translatory flight, in: J. Kelemen, P. Sosik (Eds.), Advances in Artificial Life, 6th European Conference, ECAL 2001, Prague, Czech Republic, Lecture Notes in Artificial Intelligence, Vol. 2159, Springer, Berlin, 2001, pp. 627-636.

[8] W. Reichardt, Movement perception in insects, in: W. Reichardt (Ed.), Processing of the Optical Data by Organisms and Machines, Academic Press, New York, 1969, pp. $465-493$.

[9] J. Santos-Victor, G. Sandini, F. Curotto, S. Garibaldi, Divergent stereo for robot navigation: learning from bees, in: Proceedings of the CVPR'93, 1993, pp. 434-439.

[10] M.V. Srinivasan, M. Poteser, K. Kral, Motion detection in insect orientation and navigation, Vision Research 39 (1999) 2749-2766.

[11] M.V. Srinivasan, J.S. Chahl, K. Weber, S. Venkatesh, M.G. Nagle, S.W. Zhang, Robot navigation inspired by principles of insect vision, Robotics and Autonomous Systems 26 (1999) 203-216.
[12] M.V. Srinivasan, S. Zhang, M. Altwein, J. Tautz, Honeybee navigation: nature and calibration of the "Odometer", Science 287 (2000) 851-853.

[13] M.V. Srinivasan, S.W. Zhang, J.S. Chahl, E. Barth, S. Venkatesh, How honeybees make grazing landings on flat surfaces, Biological Cybernetics 83 (2000) 171-183.

[14] S. van der Zwaan, A. Bernardino, J. Santos-Victor, Visual station keeping for floating robots in unstructured environments, Robotics and Autonomous Systems 39 (2002) 145-155.

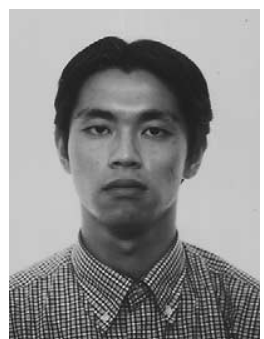

Fumiya Iida received his Master's degree in mechanical engineering from Science University of Tokyo, Japan. He is currently working as a Ph.D. student of computer science at the Artificial Intelligence Laboratory of the University of Zurich. His research interests include machine vision, human-machine interaction, and biologically inspired robotics. 